www.jmscr.igmpublication.org

Index Copernicus Value: 79.54

ISSN (e)-2347-176x ISSN (p) 2455-0450

crossrefDOI: https://dx.doi.org/10.18535/jmscr/v7i2.89

Journal Of Medical Science And Clinical Research

IGM Publication

An Official Publication of IGM Publication

\title{
Patency of Stoma after Endoscopic Dacryocystorhinostomy
}

Authors

\author{
Athira M A ${ }^{1}$, Salima Rema Windsor ${ }^{2 *}$, Satheesh $\mathbf{S}^{3}$, Rajesh $\mathbf{R}^{4}$
}

${ }^{1}$ Post graduate student, Dept of ENT, Govt. Medical College, Thiruvananthapuram

${ }^{2}$ Associate Professor, Dept of ENT, Govt. Medical College, Thiruvananthapuram

${ }^{3}$ Professor \& HOD, Dept of ENT, Govt. Medical College, Thiruvananthapuram

${ }^{4}$ Associate Professor, Dept of ENT, Govt. Medical College, Thiruvananthapuram

*Corresponding Author

Salima Rema Windsor

Associate Professor, Dept of ENT, Govt. Medical College, Thiruvananthapuram, India

\begin{abstract}
Introduction: Obstruction of the nasolacrimal duct is a common disorder manifested by epiphora, purulent discharge from the eye or swelling in the lacrimal sac area. Dacryocystorhinostomy (DCR) is a procedure performed to drain the lacrimal sac in cases of nasolacrimal duct obstruction. Endoscopic DCR has many advantages over external DCR like avoidance of scar and preservation of lacrimal pump mechanism.
\end{abstract}

Objective: The aim of this study is to evaluate the success rate of endoscopic DCR in nasolacrimal duct obstruction by studying the symptomatic relief and patency of stoma of lacrimal sac after Endoscopic DCR and to study the factors influencing the surgical outcome.

Methods: A longitudinal study which spanned a period of 18 months. All the patients who underwent Endoscopic DCR were assessed at their post operative visit, at 1 week, 2 months and 6 months after surgery. At each visit, the symptoms of the patients were noted. Stoma was assessed by syringing and endoscopic evaluation.

Results: A total of 31 patients underwent endoscopic DCR of which 25 patients had primary acquired nasolacrimal duct obstruction (PANDO). 4 cases had secondary acquired nasolacrimal duct obstruction (SANDO) following multiple facial bone fractures and 2 cases were persistent congenital nasolacrimal duct obstruction. There were 14 patients with deviated nasal septum to the same side as that of DCR, but only 3 patients warranted a concomitant septoplasty. 28 out of 31 patients had good symptomatic relief and patent healthy stoma on follow up at 6 months. 3 cases were considered as failure. 1 case underwent revision surgery and is now asymptomatic. The other 2 failed cases were advised revision surgery, but declined and preferred observation.

Conclusion: Endoscopic DCR is an effective and safe surgical procedure with high success rate in the treatment of chronic nasolacrimal duct obstruction. Relevant concomitant sinonasal pathologies should be corrected to decrease the chance of failure. Regular follow up after endoscopic DCR is necessary. Early detection of stomal problems (by syringing and nasal endoscopy) and timely intervention will lead to a successful outcome

Keywords: Nasolacrimal duct obstruction, Endoscopic Dacryocystorhinostomy (DCR), Epiphora. 


\section{Introduction}

Dacryocystorhinostomy (DCR) is a procedure performed to drain the lacrimal sac in cases of nasolacrimal duct obstruction. It can be performed externally or endoscopically. Endoscopic DCR has many advantages over external DCR. The main advantages are

1) Avoidance of facial scarring between the eye and nose.

2) No division of the medial canthal ligament

3) Preservation of the pump action of the orbicularis oculi muscle on the lacrimal sac.

4) Simultaneous treatment of other nasal pathologies

5) Reduced intraoperative bleeding.

This study was conducted to know about the surgical outcome of endoscopic dacryocystorhinostomy on short term follow up in our institution and the factors likely to influence the same. The relevance of this study is to know the effectiveness and success rate of endoscopic DCR by assessing the symptomatic relief and the patency of the stoma. In case of failure, to know the cause of failure, further modification in techniques to be done to avoid failure.

\section{Methodology}

A thorough history was taken and detailed clinical examination was done for all patients being planned for endoscopic dacryocystorhinostomy. Epiphora being the commonest symptom and present in all the patients, was graded according to Visual analogue score $(0=$ no, $1-3=$ mild, $4-6=$ moderate, $7-10=$ severe) for facilitating postoperative comparison. All the patients had undergone syringing prior to admission to confirm nasolacrimal duct as the site of block. Patients were admitted on the previous day of surgery and started on parenteral antibiotics. A written informed consent was taken. Patients were kept on empty stomach overnight. Majority of the cases were done under local anaesthesia. Few were done under general anaesthesia also. All the cases were done by experienced surgeons in the department using the standard technique employed for endoscopic dacryocystorhinostomy.

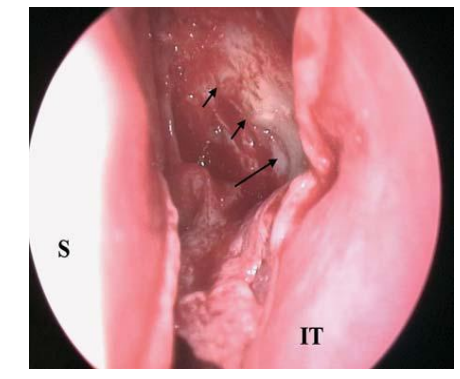

Cotton pledgets soaked in $4 \%$ lignocaine with adrenaline (1:5000) solution was placed in the nasal cavity for topical anesthesia and vasoconstriction. Mucosal incision was made in the lateral nasal wall anterior to the middle turbinate after local infiltration. Mucoperiosteal flap was elevated. The anterior lacrimal crest was identified and lacrimal bone removal was done using a Kerrison punch forceps or powered drill. Lacrimal sac was identified and a vertical slit was made on the sac. Micro scissors were then used to create an anterior and posterior flap and good mucosal approximation was obtained.

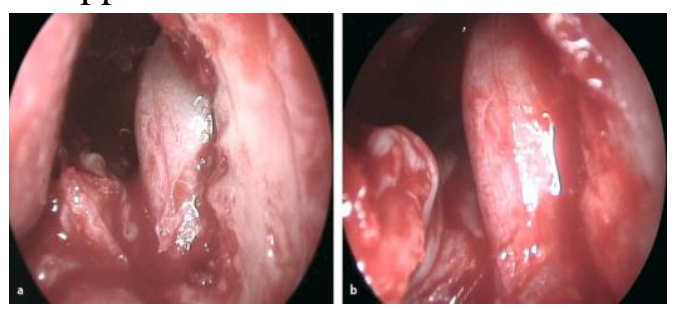

\section{Post Operative Care}

Patients were put on parenteral antibiotics, steroids, analgesics, decongestants and antibiotic eye drops. Nasal pack was removed on first post operative day and nasal douching was started. The patients were discharged on the $2^{\text {nd }}$ postoperative day. They were followed up at first week, second month and six months after surgery. At each visit the symptoms if any, were noted. Syringing and endoscopic examination of stoma was done and findings were recorded.

\section{Results}

The age of patients undergoing Endoscopic DCR in our study ranged from $5 y$ rs to $73 y$ rs. The most

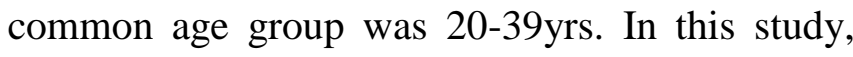


almost equal distribution of gender was observed with $51.6 \%$ males \& $48.4 \%$ females. Majority of the patients $(80.8 \%)$ had primary acquired nasolacrimal duct obstruction (PANDO). 4 $(12.8 \%)$ patients had secondary acquired nasolacrimal duct obstruction (SANDO) due to multiple facial bone fractures following RTA. 2 (6.4\%) cases had persistent congenital nasolacrimal duct obstruction. Out of 31 patients, all $(100 \%)$ had epiphora. 22 (71\%) patients had purulent discharge from the eye and 12 (9.6\%) had swelling in the lacrimal sac area. Of the patients who had epiphora, 25 patients $(80.6 \%)$ had moderate epiphora, 3 (9.7\%) had mild epiphora \& 3 (9.7\%) had severe epiphora. On preoperative syringing, all the patients had regurgitation through the upper punctum indicative of saccal or post saccal obstruction of nasolacrimal pathway. 14 patients $(45.2 \%)$ had DNS to the same side of surgery. 3 patients (9.7\%) had DNS to the opposite side of surgery. After preoperative decongestion with nasal packing, only 3 patients $(9.7 \%)$ warranted a concomitant septoplasty. Post operatively, at 6 months follow up, 28 cases $(90.3 \%)$ had no epiphora. $2(6.5 \%)$ had moderate epiphora. 1 patient $(3.2 \%)$ had severe epiphora.

On syringing at 6 months, $28(90.3 \%)$ had no regurgitation. $3(9.6 \%)$ had regurgitation through upper punctum.

Endoscopic evaluation of stomal site showed 20 $(64.5 \%)$ had ideal location of the stoma - in front $\&$ above the axilla of the middle turbinate. 8 $(25.8 \%)$ had stoma at the level of axilla of middle turbinate. In 1 case $(3.2 \%)$, the stoma was behind the axilla of the middle turbinate and in 2 cases $(6.4 \%)$, the stoma was not recognisable.

On endoscopic evaluation of stomal size, in 2 cases $(6.5 \%)$ stoma was obliterated at 6 months. 1 (3.2\%) had small stoma of $1 \times 2 \mathrm{~mm}$. Majority $(90.3 \%)$ had stomal size of $5-8 \times 3-5 \mathrm{~mm}$. Ideal stoma $>8 \times 5 \mathrm{~mm}$ described in the literature could not be found in any of our cases.

Regarding endoscopic evaluation of stomal shape, 25 cases $(80.6 \%)$ had circular or oval shape with shallow base. 3 cases $(9.6 \%)$ had circular or oval with deep base. 1case (3.2\%) had vertical slit and in 2 cases $(6.5 \%)$ stoma was not recognisable.

3 cases $(9.6 \%)$ had synechiae which was interfering the ostium, while 5cases $(16.1 \%)$ had synechiae which was not interfering the ostium \& there was no synechiae in 23 cases (74.2\%).

On endoscopic evaluation of granulations at stomal site, 25 cases $(80.6 \%)$ had no granulation at the stomal site while $6(19.4 \%)$ had granulation tissue at the stomal edges which was treated with antibiotics and local application of steroids and few of them required chemical cauterisation.

In my study, 28 cases $(90.3 \%)$ had good symptomatic relief following surgery in the 6 months follow up period. There were 3 failures in our study, attributed because being extensive synechiae leading to stomal obstruction. 1 case underwent revision surgery with concomitant septoplasty \& synechiae release. The other 2 failed cases were advised revision surgery, but declined.

\section{Conclusion}

Endoscopic DCR is an effective and safe surgical procedure with good success rate in the treatment of chronic nasolacrimal duct obstruction.

It allows simultaneous correction of associated anatomic anomalies and sino-nasal pathology, further increasing the success rate.

Syringing and regular nasal endoscopy is mandatory to assess the stoma in the postoperative period. This allows early detection of stomal problems and timely intervention leading to a successful outcome.

Circular or oval shaped stoma with site infront and above the axilla of the middle turbinate gives the best result. Even though not the ideal size,stomal size B (5-8mm X 3-5mm) too gives good results.

Synechiae $(9.6 \%)$ formation attributes to the major cause of failure following endoscopic dacryocystorhinostomy. In case of synechiae formation, early release should be advocated for good stomal patency.

Granulations if present should be taken care of. 


\section{References}

1. Wormald PJ, Kew J, Van Hasselt CA. The intranasal anatomy of the nasolacrimal sac in endoscopic dacryocystorhinostomy. Otolaryngol Head Neck Surg. 2000; 123: 307-310.

2. Linberg JV, Anderson RL, Bumsted RM, Barreras R. Study of intranasal ostium at external dacryocystorhinostomy. Arch Ophthalmol. 1982;100:1758-1762.

3. Mann BS, Wormald PJ. Endoscopic assessment of the DCR ostium after endoscopic surgery. Laryngoscope. 2006;116:1172-1174.

4. Yazici B, Yacizi Z. Final nasolacrimal ostium after external dacryocystorhinostomy. Arch Ophthalmol. 2003;121:76-80. 